\title{
Splenic Embolization Decreases Infectious Complications and Resource Utilization Compared to Splenectomy in Severely Injured Patients
}

\author{
James M. Haan, M.D. ${ }^{1}$, Grant V. Bochicchio, M.D., M.P.H. ${ }^{2}$, \\ Anne Conway, R.N., B.S.N., M.S. ${ }^{2}$, Kelly M. Bochicchio, R.N., B.S.N. ${ }^{2}$, \\ Thomas M. Scalea, M.D. ${ }^{2}$ \\ ${ }^{1}$ University of Kansas School of Medicine-Wichita Department of Surgery \\ Via Christi Hospitals, Inc. Trauma Services, Wichita, KS \\ ${ }^{2}$ University of Maryland School of Medicine Department of Surgery \\ R. Adams Cowley Shock Trauma Center, Baltimore, MD
}

\begin{abstract}
Introduction. Increasing use of main coil angioembolization for splenic injury has raised concerns of increased complication rates and resource utilization compared to splenectomy. This study examined complication rates for severely injured patients undergoing splenectomy versus main coil angioembolization.

Methods. Demographic data (age, sex, and race), Injury Severity Score (ISS), and splenic injury grade were collected prospectively on all patients admitted to the intensive care unit with blunt splenic injury treated with splenectomy or main coil angioembolization. Outcome measures (transfusion requirements, mechanical ventilation use and duration, mortality, intensive care unit and hospital length of stay, infection rate, and systemic inflammatory response syndrome or SIRS score) were reviewed daily.

Results. Of 116 patients reviewed, 65 underwent splenectomy and 51 underwent main coil angioembolization. Groups were comparable for age, sex, race, and mechanism of injury. Splenectomized patients had a higher ISS (41 vs 31) and splenic injury grade (3.7 vs 3.2). The main coil angioembolization group had a lower transfusion requirement, hospital length of stay, incidence of mechanical ventilation, nosocomial infection rate, and SIRS score. Overall, mortality and ventilator days were lower but not statistically significant.
\end{abstract}

Conclusions. Severely injured patients treated with splenectomy had significantly higher infection rates and resource utilization compared to those treated with main coil angioembolization.

KS J Med 2014; 7(1):50-57.

\section{Introduction}

Nonoperative management of splenic injury has become the standard of care in hemodynamically stable adult patients with blunt splenic injury. ${ }^{1-20}$ Some trauma centers, including our own, use splenic arteriography and embolization of vascular injuries as an adjunct to improve the success rate of splenic injuries managed nonoperatively. ${ }^{1-6,12-19}$ With the increasing use of main coil angioembolization for splenic injury, concerns have been raised of increased complication rates and resource utilization in comparison to splenectomy. ${ }^{7,20}$ However, the use of splenic embolization has been a matter of controversy for some time, where increased infectious risks from splenic artery main coil angioembolization (SA-MCE) versus splenectomy are both promoted as benefits of the procedure and an unnecessary risk. ${ }^{1,21-24}$

In light of conflicting data, many clinicians are uncertain as to the infectious risks and other complications associated with splenic artery SA-MCE. These risks 
have been addressed in patients with isolated splenic injury, but not on multi-trauma patients who presumably would be at greater risk of infection. This study examined the complication rates for multi-trauma patients undergoing splenectomy versus main splenic artery embolization regardless of hemodynamic instability.

\section{Methods}

Patient selection. Data were collected prospectively for all critically injured patients (i.e., those admitted to the intensive care unit (ICU) or intermediate care unit (IMC)) with a blunt mechanism of injury who underwent splenectomy or splenic embolization between August 2001 and August 2005. Patients who had splenic embolization followed by splenectomy were excluded from evaluation. Demographic data (age, sex, and race), injury severity score (ISS), and splenic injury grade were recorded. The grade of splenic injury was determined by computed tomography (CT) or intraoperative findings using the American Association for the Surgery of Trauma Organ Injury Scale (AAST OIS) grading system. $^{25}$

Splenic arteriography and treatment protocol. Splenic arteriography was performed on all hemodynamically stable patients with high-grade splenic injury (AAST OIS grades 3-5) and low-grade splenic injury (AAST OIS grades 1 and 2) who demonstrated active bleeding or vascular lesions on abdominal CT. .,2,12-17 $^{\text {. }}$ Unstable patients, defined as those with SBP $\leq 90$ or those the trauma attending deemed to be hemodynamically unstable, were taken directly to the operating room.

Splenic artery MCE was performed if there was evidence of pseudoaneurysm, arteriovenous fistula (AVF), or active bleeding confined to the spleen. Additionally, SA-MCE was performed if secondary evidence of vessel injury was observed (i.e., truncation) or at the discretion of the interventional radiologist and attending trauma surgeon if the patient's clinical condition warranted a more aggressive interventional approach. Selective coaxial micro-coil distal splenic artery branch embolization was performed in addition to SA-MCE if active contrast extravasation into the peritoneal cavity was noted. Patients underwent serial hematocrit determination and abdominal examinations every eight hours. When two stable examinations were obtained, patient activity was liberalized and diet begun. Follow-up abdominal CT was performed at 48 to 72 hours to rule out delayed pseudoaneurysm/ infarction with discharge if no other issues were identified. The decision between operative and non-operative management was based solely on hemodynamic stability (SBP $\geq 90$ ) and not age, associated injuries, or need for ICU admission. ${ }^{1,2,12-17}$

Outcome data. Outcome data included all-cause mortality, hospital length of stay (H-LOS), ICU length of stay (I-LOS), ventilator days, and development of infection. Infection was defined using the Centers for Disease Control (CDC) criteria where applicable; clinician judgment was applied in all other circumstances. ${ }^{26}$ The site and cultured organisms (if any) were recorded for all infections. The number of units of packed red blood cells (PRBCs) was recorded.

Statistical analysis. Analysis was performed using Stata version 8 (StataCorp, TX). Student's t-test and Pearson's chisquared analysis were used to compare continuous and categorical variables as appropriate. Multiple linear and logistic regression models were used to control for differences in demographic variables.

\section{Results}

Demographics. One hundred eighteen patients underwent splenectomy or splenic 
artery embolization. To prevent possible confounding, two patients had both procedures and were excluded from analysis, for a total of 116. Sixty-five patients (56\%) underwent splenectomy while 51 (44\%) had a splenic embolization. On univariate analysis, age, gender, and race were similar between groups, while ISS was significantly higher in the splenectomy group (40.9 vs 31.4, $\mathrm{p}=0.0002$; Table 1 ).

Table 1. Comparison of demographic data and injury severity for the splenectomy and splenic embolization patients.

\begin{tabular}{|l|c|c|c|c|c|}
\hline Treatment & Number & $\begin{array}{c}\text { Age } \\
\text { (Years) }\end{array}$ & $\begin{array}{c}\text { Gender } \\
\text { (\% Male) }\end{array}$ & $\begin{array}{c}\text { Race } \\
\text { (\% Non-white) }\end{array}$ & ISS* \\
\hline Splenectomy & $65(56 \%)$ & $42.0 \pm 18.8$ & $70.8 \%$ & $29.2 \%$ & $40.9 \pm 12.2$ \\
\hline Embolization & $51(44 \%)$ & $36.4 \pm 18.9$ & $70.6 \%$ & $23.5 \%$ & $31.4 \pm 14.7$ \\
\hline
\end{tabular}

*ISS = Injury Severity Score, $\mathrm{p}=0.0002$

Grade of splenic injury. The distribution of grades of splenic injury is given in Table 2. The distribution of grades of injury was significantly different between procedure groups, with higher grades found more commonly among patients who had a splenectomy $(p=0.01)$. Similarly, the mean splenic injury grade was significantly higher for patients who had undergone splenectomy as compared to those undergoing embolization ( $3.7 \pm 1.0$ vs $3.2 \pm 0.8$, respectively; p $=0.002)$.

Table 2. Comparison of splenic injury grade distribution by procedure.

\begin{tabular}{|c|c|c|c|c|c|c|}
\hline \multirow{2}{*}{ Treatment } & \multirow{2}{*}{ Number } & \multicolumn{5}{|c|}{ Splenic Injury Grade [\% (n)] } \\
\cline { 3 - 7 } & & 1 & 2 & 3 & 4 & 5 \\
\hline Splenectomy & 65 & $3.1 \%(2)$ & $7.7 \%(5)$ & $29.3 \%(19)$ & $38.5 \%(25)$ & $21.5 \%(14)$ \\
\hline Embolization & 51 & $3.9 \%(2)$ & $11.8 \%(6)$ & $52.9 \%(27)$ & $27.5 \%(14)$ & $3.9 \%(2)$ \\
\hline
\end{tabular}

Outcomes, complications, and mortality. On univariate analysis, patients undergoing splenectomy had a significantly greater ILOS and H-LOS than embolized patients (20.4 vs 14.2 days, $\mathrm{p}=0.05$, and 29.6 vs 15.5 days, $\mathrm{p}=0.0002$, respectively; Table 3). Similarly, patients requiring splenectomy were significantly more likely to require mechanical ventilation $(97 \%$ vs $675 \%$, p < 0.002 ), and there was a nonsignificant trend towards increased ventilator days in patients who had a splenectomy and required mechanical ventilation ( $p=0.06$; Table 3 ). Splenectomy patients also were found to have a significantly higher systemic inflammatory response syndrome (SIRS) score (2.7 vs 2.2; $\mathrm{p}<0.05$ ).

Using multiple logistic regression analysis to control for age, gender, ISS, and grade of splenic injury, embolized patients were significantly less likely to require mechanical ventilation than splenectomy patients (Odds Ratio $(\mathrm{OR})=0.14, \mathrm{p}=$ 0.028). No difference was found in the 
number of ventilator days among those requiring support $(\mathrm{p}=0.16)$. I-LOS was not different between splenectomy and embolization patients ( $\mathrm{p}=0.13$ ), however, H-LOS was significantly shorter for embolization patients (coefficient $=14.1$; $\mathrm{p}$ $=0.001$; Table 3).

On univariate analysis, mortality was $22 \%$ in the splenectomy group as compared to $9.8 \%$ in the embolization group, but this was not significant ( $p=0.09$, Table 3). Results from multiple logistic regression controlling for age, sex, ISS and grade also failed to show a significant mortality difference $(p=0.73)$.

Transfusions. On univariate analysis, splenectomy patients received significantly more units of PRBCs than embolization patients (8.5 units, Table 3). Using multiple linear regression analysis to control for age, gender, ISS, and grade of splenic injury, embolization patients received fewer units of PRBC than splenectomy patients ( $\mathrm{p}=$ 0.03).
Infection. Nearly 65\% of splenectomy patients developed an infection during hospitalization $(n=42)$ while $39.2 \%$ of embolization patients developed infection ( $p$ $=0.0006$; Table 3). The unadjusted relative risk of infection was 0.61 for embolization patients as compared to splenectomy patients. Logistic regression analysis controlling for age, gender, ISS and grade of splenic injury yielded an OR of 0.35 for infection in embolization patients $(\mathrm{p}=$ $0.022)$.

To determine if this effect was due to the higher transfusion requirement and/or greater incidence of hypotension in the group undergoing splenectomy, or related purely to the treatment technique of embolization and splenic conservation, data were reexamined using linear regression controlling for these variables. Initial hypotension had no effect on outcome variables. When controlled for transfusion requirements, however, only reduced $\mathrm{H}$ LOS for MCE remained statistically significant $(p<0.05)$.

Table 3. Comparison of outcome variables for patients undergoing splenectomy (SPL) vs. main coil embolization (MCE).

\begin{tabular}{|l|c|c|c|c|c|c|c|c|}
\hline Treatment & I-LOS & H-LOS & Vent \% & $\begin{array}{c}\text { Vent } \\
\text { days }\end{array}$ & PRBC & $\begin{array}{c}\text { NOS } \\
\text { Infect }\end{array}$ & SIRS & Mortality \\
\hline SPL & 20.4 & 29.6 days & $97 \%$ & 22 days & $14.5 \mathrm{u}$ & $64.6 \%$ & 2.7 & $22.0 \%$ \\
\hline MCE & 14.2 & 15.5 days & $67 \%$ & 15 days & $6.0 \mathrm{u}$ & $39.2 \%$ & 2.2 & $9.8 \%$ \\
\hline P value & 0.05 & 0.0002 & $<0.001$ & 0.06 & 0.03 & 0.0006 & $<0.05$ & 0.09 \\
\hline
\end{tabular}

I-LOS = ICU length of stay, H-LOS = hospital length of stay, Vent $\%=$ percent requiring mechanical ventilation, Vent days $=$ days on mechanical ventilation, $\mathrm{PRBC}=$ packed blood cell transfusion requirements, NOS Infect $=$ percent with Nosocomial Infection, SIRS $=$ systemic inflammatory response syndrome.

The most commonly cultured organism in embolization patients was Staphylococcus aureus; the most common organism in splenectomy patients was Pseudomonas aeruginosa. Splenectomy patients developed significantly more abdominal, respiratory, bloodstream, and urinary tract infections than embolization patients (Figure 1), however, the overall distribution of infection sites was approximately similar between the two groups. Only seven infections involved encapsulated bacteria; three S. pneumoniae (one SA-MCE, two splenectomy) and four $H$. influenzae (two SA-MCE, two 
splenectomy). There was one S. pneumoniae intra-abdominal abscess in the splenectomy group with the remainder being respiratory in origin, with no mortality directly attributed to any of these infections.

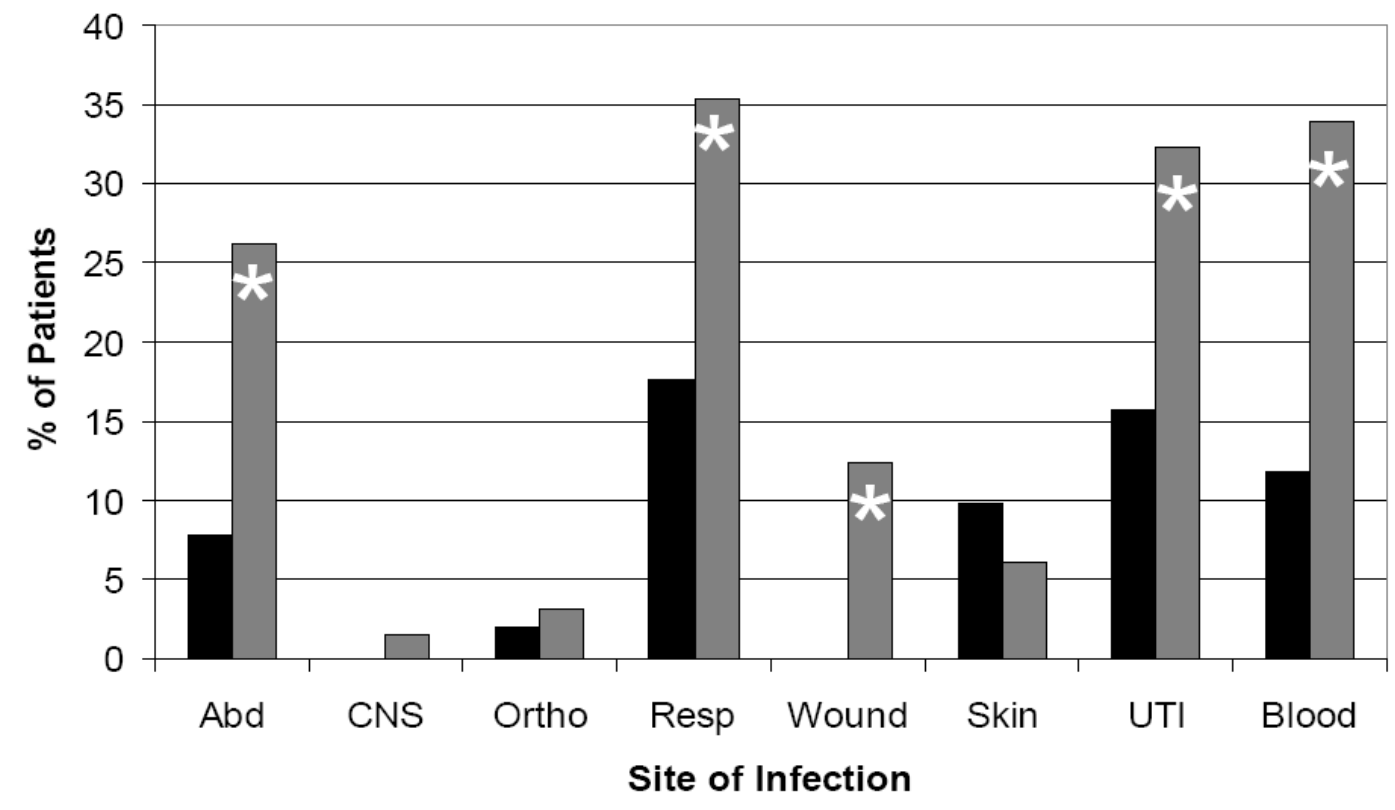

Embolization $\square$ Splenectomy

Figure 1. Percentage of patients developing infection at a given site.

\section{Discussion}

There remains some debate regarding the utility of splenic angioembolization. A few authors feel that there is no improvement to the rate of splenic gland preservation from angioembolization. ${ }^{7,20} \mathrm{~A}$ larger group has voiced concerns regarding post-procedure complication rates, patient outcomes, and the unknown immunologic effects stemming from splenic embolization. $^{1-20}$ Prior studies have documented the complication rates of angioembolization and our study documented low procedural complication rates, even in the most severely injured patients. ${ }^{1-6,12-19}$ There were significantly improved outcomes regarding $\mathrm{H}$-LOS, need for mechanical ventilation, need for transfusion, and mortality.
A limitation of this study was that the splenectomy group was comprised of unstable patients while embolization patients were stable. Although hypotension had no effect on outcome, those patients with increased transfusions requirements had worse outcomes regardless of treatment arm. The transfusions seemed to lead to increased duration of mechanical ventilation, hospital stay, and mortality. This was not surprising as infections and acute respiratory distress syndrome (ARDS) are known to be related to patient transfusion. Numerous researchers have demonstrated similar relationships between blood transfusions and the prolongation of H-LOS, the development of multisystem organ failure, or the incidence of either infection or mortality. ${ }^{27-30}$ 
In a prospective study, 102 consecutive patients with severe trauma that required blood transfusion were followed. ${ }^{31}$ Acute exposure to transfused blood led to a higher rate of ARDS with $21 \%$ of patients who received 0 to 5 units of PRBCs, 31\% of those patients who received 6 to 10 units of PRBCs, and $57 \%$ of those who received greater than 10 units of PRBCs developing ARDS ( $<<0.007)$. The association between the amount of transfused blood and the development of ARDS remained significant in their multivariable logistic regression model. This demonstrated the amount of transfused blood is associated independently with both the development of ARDS and hospital mortality. The age of blood also impacted the SIRS response, ${ }^{32,33}$ and as a tertiary center older blood generally is received for use. Therefore, the improved outcomes of SA-MCE patients in this study may be related directly to decreased transfusion requirements associated with SA-MCE or, more likely, a selection bias of the more stable patients with lower preexisting blood loss undergoing SA-MCE.

The immunologic effects of splenic embolization remain poorly defined. Pirasteh and coworkers demonstrated encouraging results regarding splenic function based on absence of Howell-Jolly bodies on peripheral smear and normal uptake on technetium-99 scan. $^{34}$ Bessoud et al. ${ }^{19}$

\section{References}

${ }^{1}$ Haan JM, Bochicchio GV, Kramer N, Scalea TM. Nonoperative management of blunt splenic injury: A 5-year experience. J Trauma 2005; 58(3):492-498. PMID: 15761342.

2 Shanmuganathan K, Mirvis SE, BoydKranis R, Takada T, Scalea TM. Nonsurgical management of blunt splenic injury: Use of CT criteria to select patients for splenic arteriography and potential demonstrated normal exposure driven immunity, streptococcal vaccine response, and splenic Doppler flow in the majority of patients following main coil embolization. This was limited to twenty-four patients evaluated with Doppler and exposure titers. Only six patients underwent immunization with one failure in a patient with chronic lymphocytic leukemia. None of the prior studies focused on clinical evidence of infection. Our study was focused on the most severely injured patients, all of which required ICU or IMC admission. The theory was that this cohort had the highest infection risks and rates. If there were some elements of early immunosuppression related to embolization, this cohort would be more likely to demonstrate this with a higher infection rate than the patients with isolated splenic injury. The fact that the infection and resource utilization rate were lower for the embolization cohort at a minimum was reassuring.

In conclusion, splenic embolization remains a valuable adjunct in splenic salvage. Severely injured patients treated with splenectomy had significantly higher infection rates and resource utilization rates when compared to those treated with main coil embolization. The major correlation with negative results was patient transfusion requirements.

endovascular therapy. Radiology 2000; 217(1):75-82. PMID: 11012426.

3 Federle MP, Courcoulas AP, Powell M, Ferris JV, Peitzman AB. Blunt splenic injury in adults: Clinical and CT criteria for management, with emphasis on active extravasation. Radiology 1998; 206(1): 137-142. PMID: 9423663.

4 Sclafani SJ, Shaftan GW, Scalea TM, et al. Nonoperative salvage of computed tomography-diagnosed splenic injuries: 
Utilization of angiography for triage and embolization for hemostasis. J Trauma 1995; 39(5):818-827. PMID: 7473996.

${ }^{5}$ Davis KA, Fabian TC, Croce MA, et al. Improved success in nonoperative management of blunt splenic injuries: Embolization of splenic artery pseudoaneurysms. J Trauma 1998; 44(6):10081013. PMID: 9637156.

${ }^{6}$ Bee TK, Croce MA, Miller PR, Pritchard FE, Fabian TC. Failures of splenic nonoperative management: Is the glass half empty or half full? J Trauma 2001; 50(2):230-236. PMID: 11242286.

7 Peitzman AB, Heil B, Rivera L, et al. Blunt splenic injury in adults: Multiinstitutional Study of the Eastern Association for the Surgery of Trauma. J Trauma 2000; 49(2):177-189. PMID: 10963527.

8 Becker CD, Spring P, Glättli A, Schweizer W. Blunt splenic trauma in adults: Can CT findings be used to determine the need for surgery? Am J Roentgenol 1994; 162(2):343-347. PMID: 8310923.

${ }^{9}$ Kohn JS, Clark DE, Isler RJ, Pope CF. Is CT tomographic grading of splenic injury useful in the nonsurgical management of blunt trauma? J Trauma 1994; 36(3):385390. PMID: 8145321.

${ }^{10}$ Schurr MJ, Fabian TC, Gavant ML, et al. Management of blunt splenic trauma: Computed tomographic contrast blush predicts failure of nonoperative management. J Trauma 1995; 39(3):507-513. PMID: 7473916.

${ }^{11}$ Gavant ML, Schurr MJ, Flick PA, Croce MA, Fabian TC, Gold RE. Predicting clinical outcome of nonsurgical management of blunt splenic injury: Using CT to reveal abnormalities of splenic vasculature. AJR Am J Roentgenol 1997; 168(1):207-212. PMID: 8976947.

${ }^{12}$ Haan JM, Biffl W, Knudson MM, et al. Splenic embolization revisited: A multicenter review. J Trauma 2004; 56(3):542-547. PMID: 15128125.

${ }^{13}$ Haan JM, Mamery H, Shanmuganathan K, Mirvis SE, Scalea TM. Experience with splenic main coil embolization and significance of new or persistent pseudoaneurysm: Reembolize, operate, or observe. J Trauma 2007; 63(3):615-619. PMID: 18073609.

${ }^{14}$ Haan J, Scott J, Boyd-Kranis RL, Ho S, Kramer M, Scalea TM. Admission angiography for blunt splenic injury: Advantages and pitfalls. J Trauma 2001; 51(6):1161-1165. PMID: 11740269.

${ }^{15}$ Haan J. The management of blunt splenic injury: Observe, embolize, or operate? Trauma Quarterly 2002; 15:27-43.

${ }^{16}$ Haan JM, Boswell S, Stein D, Scalea TM. Follow-up abdominal CT is not necessary in low-grade splenic injury. Am Surg 2007; 73(1):13-18. PMID: 17249449.

${ }^{17}$ Shanmuganathan K, Mirvis SE, Sover ER. Value of contrast-enhanced CT in detecting active hemorrhage in patients with blunt abdominal or pelvic trauma. AJR Am J Roentgenol 1993; 161(1):6569. PMID: 8517323

${ }^{18}$ Omert LA, Salyer D, Dunham CM, Porter J, Silva A, Protetch J. Implications of the "contrast blush" finding on computed tomographic scan of the spleen in trauma. J Trauma 2001; 51(2):272-278. PMID: 11493784.

${ }^{19}$ Bessoud B, Duchosal MA, Siegrist CA, et al. Proximal splenic artery embolization for blunt splenic injury: Clinical, immunologic, and ultrasound-Doppler follow-up. J Trauma 2007; 62(6):14811486. PMID: 17563670.

${ }^{20}$ Harbrecht BG, Ko SH, Watson GA, Forsythe RM, Rosengart MR, Peitzman AB. Angiography for blunt splenic trauma does not improve the success rate of nonoperative management. J Trauma 2007; 63(1):44-49. PMID: 17622867. 
${ }^{21}$ Demetriades D, Scalea TM, Degiannis E, et al. Blunt splenic trauma: Splenectomy increases early infectious complications: a prospective multicenter study. J Trauma Acute Care Surg 2012; 72(1):229-234. PMID: 22310131.

${ }^{22}$ Harbrecht BG, Ko SH, Watson GA, Forsythe RM, Rosengart MR, Peitzman AB. Angiography for blunt splenic trauma does not improve success rate of nonoperative management. J Trauma 2007; 63(1):44-49. PMID: 17622867.

${ }^{23}$ Haan JM, Biffl W, Knudson MM, et al. Splenic embolization revisited: A multicenter review. J Trauma 2004; 56(3):542-547. PMID: 15128125.

${ }^{24}$ Peitzman AB, Ferrada P, Puyana JC. Nonoperative management of blunt abdominal trauma: Have we gone too far? Surg Infect (Larchmt) 2009; 10(5):427433. PMID: 19860573.

${ }^{25}$ American Association for the Surgery of Trauma. Splenic Injury Grading Scale. http://www.aast.org/Library/TraumaTools/ InjuryScoringScales.aspx\#spleen.

Accessed September 9, 2013.

${ }^{26}$ Centers for Disease Control and Prevention. CDC/NHSN Surveillance Definitions for Specific Types of Infections. http://www.cdc.gov/nhsn/pdfs/ pscmanual/17pscnosinfdef_current.pdf.

Accessed January 20, 2014.

${ }^{27}$ Taylor RW, Manganaro L, O’Brien J, Trottier SJ, Parkar N, Veremakis C. Impact of allogenic packed red blood cell transfusion on nosocomial infection rates in the critically ill patient. Crit Care Med 2002; 30(10):2249-2254. PMID: 12394952.

${ }^{28}$ Moore FA, Moore EE, Sauaia A. Blood transfusion. An independent risk factor for postinjury multiple organ failure. Arch
Surg 1997; 132(6):620-625. PMID: 9197854.

${ }^{29}$ Robinson WP $3^{\text {rd }}$, Ahn J, Stiffler A, et al. Blood transfusion is an independent predictor of increased mortality in nonoperatively managed blunt hepatic and splenic injuries. J Trauma 2005; 58(3): 437-445. PMID: 15761334.

${ }^{30}$ Claridge JA, Sawyer RG, Schulman AM, McLemore EC, Young JS. Blood transfusions correlate with infections in trauma patients in a dose-dependent manner. Am Surg 2002; 68(7):566-572. PMID: 12132734.

${ }^{31}$ Silverboard H, Aisiku I, Martin GS, Adams M, Rozycki G, Moss M. The role of acute blood transfusion in the development of acute respiratory distress syndrome in patients with severe trauma. J Trauma 2005; 59(3):717-723. PMID: 16361918.

${ }^{32}$ Zallen G, Offner PJ, Moore EE, et al. Age of transfused blood is an independent risk factor for postinjury multiple organ failure. Am J Surg 1999; 178(6):570-572. PMID: 10670874.

${ }^{33}$ Purdy FR, Tweeddale MG, Merrick PM. Association of mortality with age of blood transfused in septic ICU patients. Can J Anaesth 1997; 44(12):1256-1261. PMID: 9429042.

${ }^{34}$ Pirasteh A, Snyder LL, Lin R, et al. Temporal assessment of splenic function in patients who have undergone percutaneous image-guided splenic artery embolization in the setting of trauma. J Vasc Interv Radiol 2012; 23(1):80-82. PMID: 22030457.

Keywords: spleen/injuries, splenectomy, therapeutic embolization, infection/ complications 